\title{
Reconstrucción filogenética de Sphaeriidae (Veneroida: Bivalvia) y la posición filogenética de Pisidium chilense
}

\section{Phylogenetic reconstruction of Sphaeriidae (Veneroida: Bivalvia) and phylogenetic position of Pisidium chilense}

\author{
Carmen Fuentealba-Jara', Guillermo D’Elia², Fidelina González³ y Cristian Franco-Jaccard ${ }^{4}$
}

\begin{abstract}
1Instituto de Ciencias Naturales, Universidad de las Américas. Campus el Boldal, Av. Jorge Alessandri № 1160 Concepción, Bío-Bío, Chile. 2Instituto de Ciencias Ambientales y Evolutivas, Facultad de Ciencias, Universidad Austral de Chile. Campus Isla Teja s/n, Valdivia, Los Ríos, 5090000, Chile ${ }_{3}^{3}$ Departamento de Biología Celular, Facultad de Ciencias Biológicas Universidad de Concepción. Campus Concepción, Barrio Universitario, Concepción, Bío-Bío, 4070386, Chile ${ }^{4}$ Departamento de Geofísica, Facultad de Ciencias Físicas y Matemáticas, Universidad de Concepción. Campus Concepción, Av. Esteban S. Iturra s/n, Barrio Universitario, Concepción, Bío-Bío, 4070386, Chile e-mail: cfuentea@udec.c
\end{abstract}

Recibido: 27 de enero de 2015.

Aceptado: 28 de julio de 2016.

Fuentealba-Jara, C., G. D’Elia, F. González y C. Franco-Jaccard. 2017. Reconstrucción filogenética de Sphaeriidae (Veneroida: Bivalvia) y la posición filogenética de Pisidium chilense. Hidrobiológica 27(1): 115-117. D0l: 10.24275/uam/izt/dcbs/hidro/2017v27n1/DElia

\section{RESUMEN}

Antecedentes. La subfamilia Sphaeriinaede bivalvos de agua dulce está formado por tres géneros, Musculium, Pisidium y Sphaerium. Sin embargo, la monofilia de los dos últimos ha sido cuestionada en diferentes análisis filogenéticos. Objetivos. En este estudio se incorpora al análisis filogenético a Pisidiumchilense, basado en secuencias del espaciador interno (ITS-1) nuclear ribosomal. Métodos. La matriz de datos se analizó bajo inferencia bayesiana y máxima parsimonia. Los resultados apoyan la monofilia de Sphaeriinae. Resultados. Los géneros Pisidium y Musculium son monofiléticos, mientras Sphaerium es parafilético respecto a Musculium. Al interior de Pisidium, las especies de América del Sur, y únicos representantes del subgénero Afropisidium incluido en el análisis, forman un grupo monofilético. Conclusiones. Antes de proponer escenarios biogeográficos o taxonómicos para Sphaeriinae es necesario realizar un muestreo taxonómico más intenso, al igual que ampliar el muestreo de caracteres para incluir loci nucleares. Palabras clave: Afropisidium, Chile, Musculium, Sphaeriidae, Sphaerium.

Palabras clave: Afropisidium, Chile, Musculium, Sphaeriidae, Sphaerium.

\section{ABSTRACT}

Background. The subfamily Sphaeriinae of freshwater bivalves is formed by three genera, Musculium, Pisidium,and Sphaerium. However, the monophyly of the latter two has been questioned in different phylogenetic analyses. Goals. In this study, we added Pisidiumchilenseto the phylogenetic analysis, based on sequences of nuclear ribosomal, an internal transcribed spacer(ITS-1). Methods. The data matrix was analyzed with maximum parsimony and Bayesian inference. Results support the monophyly of Sphaeriinae. The genera Pisidium and Musculium are monophyletic, while Sphaerium is paraphyletic with respect to Musculium. Within Pisidium, the South American species and sole representatives included in this analysis of the subgenus Afropisidium formed a monophyletic group. Conclusions. Before advancing new biogeographic or taxonomic scenarios for Sphaeriinae, further analyses based on denser taxonomic and character sampling are needed.

Key words: Afropisidium, Chile, Musculium, Sphaeriidae, Sphaerium.

La familia Sphaeriidae de almejas "uña de dedo" se distribuye en Chile entre los $18^{\circ} \mathrm{S}$ y $56^{\circ} \mathrm{S}$ y habita en una gran variedad de ecosistemas dulceacuícolas (Fuentealba et al., 2010). Actualmente, en Chile se reconoce un total de once especies, incluidas en los géneros Sphaerium (2 spp.), Musculium (2 spp.) y Pisidium (7 spp.). A pesar de que en los últimos años el conocimiento sobre los Sphaeriidae de Chile ha presentado avances considerables (e.g., Sobarzo et al., 2002; Ituarte, 2007; Parada et al., 2009; Jara-Sequel et al., 2010, 2011), la familia aún no ha sido estudiada filogenéticamente. Los estudios filogenéticos de Sphaeriidae (e.g., Lee \& ÓFoighil, 2003) han incorporado sólo a dos representantes sudamericanos, uno de Argentina y otro de Ecuador.

El presente estudio propone hipótesis filogenéticas para Sphaeriidae (basadas en caracteres moleculares), en las cuales se incorpora la especie endémica de Chile Pisidium chilense (d'Orbigny, 1846). Este muestreo taxonómico permite poner a prueba la monofilia de los géneros Musculium, Pisidium y Sphaerium, clásicamente reconocidos en la subfamilia Sphaeriidae. 
Los análisis realizados se basaron en secuencias nucleotídicas del gen nuclear transcrito interno (ITS-I). Se incluyeron 42 especies de los géneros Musculium, Pisidium y Sphaerium, obtenidas de la base de datos del Genbank. Además, se incluyó la muestra de Pisidium chilense, colectada en la Laguna Chica de San Pedro, Región del Bio-Bío, Chile

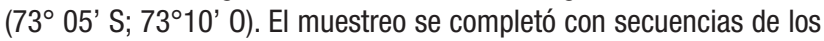
euperinos Eupera cubensis y E. platensis, que se usaron para conformar el grupo externo. En la Fig. 1 se indican los detalles de las secuencias analizadas. La reconstrucción filogenética se realizó mediante Máxima Parsimonia (MP) (Farris, 1982) e Inferencia Bayesiana (IB) (Rannala \& Yang, 1996). El análisis de MP se realizó en el programa PAUP* 4.0b10 Swofford (2000), por medio de la interfaz gráfica PaupUp (Calendini \&
Martín, 2005), con una búsqueda heurística (500 réplicas de adición de secuencias al azar) y el algoritmo de reconexión de ramas TBR. El soporte de los nodos fue evaluado mediante un análisis de bootstrap con 1000 réplicas. El análisis de IB se implementó en Bayes Phylogenies v 1.0 (Pagel \& Meade, 2004), basado en el modelo General de Tiempo Reversible (GTR) (Rodríguez et al., 1990). Fueron generadas nueve cadenas independientes (se obtuvieron aproximadamente 100'000,000 de árboles filogenéticos), muestreando cada 10,000 árboles para asegurar la independencia de los muestreos. El programa Bayes Trees 1.0 (Meade \& Pagel, 2009) se utilizó para visualizar los árboles y estimar los valores de probabilidad posterior bayesiana (PPB).

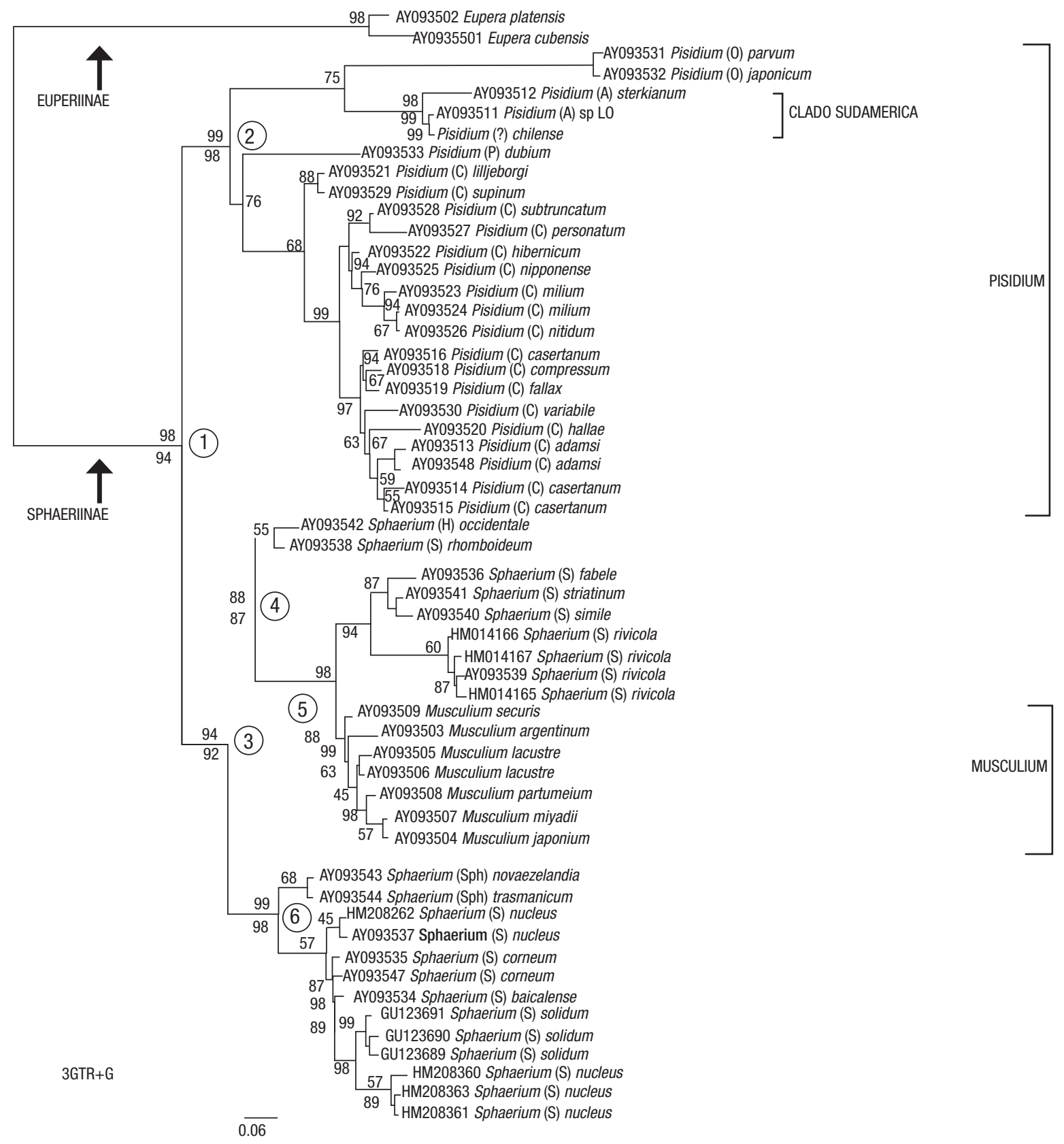

Figura 1. Árbol bayesiano consensuado por regla de la mayoría de Sphaeriidae. Sobre los nodos se muestran los valores de probabilidad a posteriori (PP) y bajo los nodos los valores de bootstrap (BS). Los círculos corresponden a los nodos de interés. Las flechas indican las subfamilias de Sphaeriidae. 
En los dos análisis filogenéticos realizados se recuperó la misma topología (Fig. 1). Sphaeriidae es monofilética (PPB = 98, BS =94), lo que es consistente con las reconstrucciones filogenéticas basadas en caracteres morfológicos (Dreher-Mansur \& Meier-Brook, 2000; Korniushin \& Glaubrecht, 2002, 2006) y en secuencias nucleares y mitocondriales (Cooley \& ÓFoighil, 2000; Lee \& ÓFoighil, 2003). Los géneros Musculium (PPB $=99, \mathrm{BS}=98$ ) y Pisidium $(\mathrm{PP}=88, \mathrm{BS}=99$ ) resultaron monofiléticos, y el género Sphaerium es parafilético respecto a Musculium. Cooley y 0' Foighil (2000) y Lee y O'Foighil (2003) sólo recuperaron como monofilético a Musculium (anidado dentro de Sphaerium), lo que sugiere que Musculium sea reconocido como un subgénero de Sphaerium.

Las especies de Sphaerium forman cuatro clados principales, correspondientes a los subgéneros Amesoda, Herringtonium, Sphaerinovay Sphaerium, los que se relacionan entre sí y con Musculium de la siguiente forma: (Sphaerium, Sphaerinova) (Herringtonium (Musculium, Amesoda)). Esta topología sugiere que se deberían formalizar cambios taxonómicos, elevar a género los actuales subgéneros de Sphaerium o subsumir (como fue sugerido por Lee y 0'Foighil, 2003) a Musculium bajo Sphaerium, lo que permite reconocer cinco subgéneros al interior del último.

La incorporación de Pisidium chilense en el análisis, permitió recuperar con fuerte soporte la monofilia de Pisidium s.I. Esto no invalida el uso de los géneros Afropisidium, Cyclocalyx, Odhneripisidium y Pisidium s.s., ni tampoco su reconocimiento como subgéneros de Pisidium, ya que el presente análisis también los recupera como monofiléticos. De igual forma, se recupera con fuerte apoyo un clado integrado por las tres especies sudamericanas de Pisidium incluidas en el análisis: P. chilense de Chile, P. sterkianum Pilsbry, 1897de Argentina y Pisidium sp. de Ecuador; i.e., el clado Afropisidium. Sin embargo, antes de proponer escenarios biogeográficos o taxonómicos para Sphaeriidae es necesario realizar un muestreo taxonómico más intenso y ampliar el muestreo de caracteres para incluir loci nucleares.

\section{AGRADECIMIENTOS}

Este estudio fue parcialmente financiado por FONDECYT 1141055.

\section{REFERENCIAS}

Calendini, F. \& J. F. Martin. 2005. Paup UP versión, 1.0.3.1. A free graphical frontend for PAUP* software. Available at: http://www.agromontpellier.fr/sppe/Recherche/JFM/PaupUp (downloaded January $5,2015)$.

Cooley, L. R. \& D. O. Folghil. 2000. Phylogenetic analysis of the Sphaeriidae (Mollusca: Bivalvia) based on partial mitochondrial 16S rDNA gene sequences. Invertebrate Biology 119: 299-308. D0I: 10.1111/ j.1744-7410.2000.tb00016.x

Dreher-Mansur, M. C. \& C. Meier-Brook. 2000. Morphology of Eupera Bourguignat, 1854 and Byssanodonta Orbigny, 1846 with contributions to the phylogenetic systematics of Sphaeriidae and Corbiculidae (Bivalvia, Veneroida). Archivfür Molluskenkunde 128: 1-59.

FarRIS, J. S. 1982.The logical basis of phylogenetic analysis. In: Platnick, N. \& V. Funk, (Eds.). Advances in Cladistics: Proceedings of the Second Meeting of the Willi Hennig Society. Columbia University Press, New York, USA, pp. 7-36.
Fuentealba, C., J. J. Morrone \& R. Figueroa. 2010. Análisis de endemismo de moluscos dulceacuícolas de Chile. Revista Chilena de Historia Natural 83: 289-298. D0I: 10.4067/S0716-078X2010000200009

ItUARTE, C. 2007. Las especies de Pisidium Pfeiffer de Argentina, Bolivia, Chile, Perú y Uruguay (Bivalvia-Sphaeriidae). Revista del Museo Argentino de Ciencias Naturales 9 (2): 169-203.

Jara-Seguel, P., E. Parada, S. Peredo, C. Palma-Rojas \& E. Von Brand. 2010. Nuclear DNA content in two chilean species of Pisidium (Veneroida: Sphaeriidae). Journal of Shellfish Research 29 (1): 101-106. D0I: 10.2983/035.029.0102

Jara-Seguel, P., S. Peredo, E. Von Brand \& E. Parada. 2011. Some aspects of the reproductive biology of two populations of Musculium argentinum (D'Orbigny, 1835) (Bivalvia: Sphaeriidae) from southern Chile. Journal of Shellfish Research 30: (2): 287-294. D0I: $10.2983 / 035.030 .0214$

Korniushin, A. V. \& M. Glaubrecht. 2002. Phylogenetic analysis based on the morphology of viviparous freshwater clams of the family Sphaeriidae (Mollusca, Bivalvia, Veneroida). Zoologica Scripta 31 (5): 415-459. DOI: 10.1046/j.1463-6409.2002.00083.x

KorNIUSHin, A. \& M. GLAUBREEHt. 2006. Anatomy and reproduction of viviparous Pisidium (Parapisidium) reticulatum Kuiper, 1966: implications for the phylogeny of Sphaeriidae (Mollusca: Bivalvia: Heterodonta). Organism, Diversity and Evolution 6: 185-195. D0I: 10.1016/j. ode.2005.09.003

LEE, T. \& D. Ó FolGHIL. 2003. Phylogenetic structure of the Sphaeriinae, a global clade of freshwater bivalve molluscs, inferred from nuclear (ITS-1) and mitochondrial (16S) ribosomal gene sequences. Zoological Journal of the Linnean Society 137: 245-260. DOI: 10.1046/j.1096-3642.2003.00047.x

Meade, A. \& M. Pagel. 2009. Software Bayes trees V.1.3. Avalaible on line at: http://www.evolution.reading.ac.uk/BayesTrees. (downloaded February 25, 2012).

Pagel, M. \& A. Meade. 2004. A phylogenetic mixture model for detecting pattern-heterogeneity in gene sequence or character-state data. Systematic Biology 53: 571-581. DOI: 10.1080/10635150490468675

Parada, E., S. Peredo \& P. Jara-Seguel. 2009. Registro actual de especies del genero Musculium link, 1807 (Bivalvia: Sphaeriidae) en chile con notas sobre morfologia y biometria de sus poblaciones. Gayana 73:49-56. DOl: 10.4067/S0717-65382009000100008

Rannala, B. \& Z. Yang. 1996. Probability distribution of molecular evolutionary trees: a new method of phylogenetic inference. Journal of Molecular Evolution 43: 304-311. D0I: 10.1007/BF02338839

Rodriguez, F., J. F. Oliver, A. Marin \& J. R. Medina. 1990. The general stochastic model of nucleotide substitution. Journal of Theoretical Biology 142: 485-501. D0I: 10.1016/S0022-5193(05)80104-3.

Sobarzo, C., P. Jara-Seguel, S. Peredo \& E. Parada. 2002. Primer registro de Musculium argentinum (d' Orbigny, 1835) (Bivalvia: Sphaeriidae) en aguas continentales chilenas. Gayana Zoologia 66 (1): 39-43. D0I: 10.4067/S0717-65382002000100006

Swofford, D. 2000. PAUP: Phylogenetic Analysis Using Parsimony (and other Methods), version 4.0.Sinauer Associates Inc., Sunderland, MA, USA. 